\title{
Functional Genomic Analysis of Remyelination Reveals Importance of Inflammation in Oligodendrocyte Regeneration
}

\author{
Heather A. Arnett, ${ }^{1,2 \star}$ Ying Wang, ${ }^{1,3 \star}$ Glenn K. Matsushima, ${ }^{2,3}$ Kinuko Suzuki, ${ }^{2,4}$ and Jenny P.-Y. Ting ${ }^{1,2,3}$ \\ ${ }^{1}$ Lineberger Comprehensive Cancer Center, ${ }^{2}$ Neuroscience Center, and Departments of ${ }^{3}$ Microbiology-Immunology and ${ }^{4}$ Pathology and Laboratory \\ Medicine, University of North Carolina, Chapel Hill, North Carolina 27599
}

Tumor necrosis factor $\alpha(\mathrm{TNF} \alpha)$, a proinflammatory cytokine, was shown previously to promote remyelination and oligodendrocyte precursor proliferation in a murine model for demyelination and remyelination. We used Affymetrix microarrays in this study to identify (1) changes in gene expression that accompany demyelination versus remyelination and (2) changes in gene expression during the successful remyelination of wild-type mice versus the unsuccessful attempts in mice lacking TNF $\alpha$. Alterations in inflammatory genes represented the most prominent changes, with major histocompatibility complex (MHC) genes dramatically enhanced in microglia and astrocytes during demyelination, remyelination, and as a consequence of TNF $\alpha$ stimulation. Studies to examine the roles of these genes in remyelination were then performed using mice lacking specific genes identified by the microarray. Analysis of MHC-II-null mice showed delayed remyelination and regeneration of oligodendrocytes, whereas removal of MHC-I had little effect. These data point to the induction of MHC-II by TNF $\alpha$ as an important regulatory event in remyelination and emphasize the active inflammatory response in regeneration after pathology in the brain.

Key words: oligodendrocytes; glia; neuroinflammation; regeneration; major histocompatibility complex; gene array; multiple sclerosis; remyelination; demyelination; TNF

\section{Introduction}

Inflammation in the CNS is thought to be an exacerbating factor for many neurodegenerative and demyelinating disorders, most notably multiple sclerosis (MS). Many products of inflammation are seen in the plaques of patients with MS and have been proposed to contribute to the destruction of white matter (Brosnan and Raine, 1996; Sriram and Rodriguez, 1997; Raine et al., 1998); however, a protective role for neuroinflammation and inflammatory cytokines such as tumor necrosis factor $\alpha(\mathrm{TNF} \alpha)$ has emerged recently in models of demyelination and traumatic brain injury (Eugster et al., 1999; Scherbel et al., 1999; Suvannavejh et al., 2000; Juedes and Ruddle, 2001; Arnett et al., 2002). In addition, the deletion of TNF $\alpha$ in mice caused susceptibility to an immune-mediated demyelination model (Korner et al., 1997; Liu et al., 1998; Kassiotis et al., 1999). The ability of TNF $\alpha$ to generate multiple and sometimes opposing effects (exacerbatory, protective, or regenerative) can be attributed in part to the presence of its two receptors, TNFR1(p55) and TNFR2(p75) (Locksley et al., 2001). In an autoimmune demyelinating model, TNFR1 has been shown to be involved primarily in the initiation of demyelination, whereas TNFR2 often appears either uninvolved or yields a protective effect (Eugster et al., 1999; Suvan-

\footnotetext{
Received March 31, 2003; revised July 25, 2003; accepted July 29, 2003.

This work was supported by National Institutes of Health Grants NS34190 (J.P.-Y.T.) and NS24453 (K.S.) and National Multiple Sclerosis Society Grant RG1785 (J.P.-Y.T.).

*H.A.A. and Y.W. contributed equally to this work.

Correspondence should be addressed to Heather A. Arnett, Dana-Farber Cancer Institute, Smith Building 1070, Harvard Medical School, One Jimmy Fund Way, Boston, MA 02115. E-mail: heather_arnett@dfci.harvard.edu. Copyright $\odot 2003$ Society for Neuroscience $\quad$ 0270-6474/03/239824-09\$15.00/0
}

navejh et al., 2000; Kassiotis and Kollias, 2001). Using a neurotoxicant (cuprizone) to induce demyelination and study remyelination, we demonstrated previously that TNF $\alpha$ promotes remyelination and oligodendrocyte regeneration through TNFR2 (Arnett et al., 2001).

In agreement with these preclinical studies implicating TNF $\alpha$ in the amelioration of demyelinating disease, the use of anti-TNF therapy in patients with MS caused disease exacerbation, and its use in patients with rheumatoid arthritis resulted in new demyelinating lesions and new-onset MS (Lenercept Group, 1999; Mohan et al., 2001; Robinson et al., 2001; Sicotte and Voskuhl, 2001). A better understanding of the observed ability of TNF $\alpha$ to promote remyelination may lead to effective therapeutic interventions in the treatment of demyelinating diseases. Furthermore, because TNF $\alpha$-null mice are defective in their ability to remyelinate, they provide a unique model for identifying factors important to this process.

To analyze global changes that occur during demyelination, remyelination, and $\mathrm{TNF} \alpha$-directed remyelination in the cuprizone model, we now use Affymetrix cDNA microarrays to identify (1) genes that accompany demyelination versus remyelination and (2) gene differences in wild-type and $\mathrm{TNF} \alpha$-null to compare successful versus unsuccessful remyelination. The latter are especially interesting because they should include differences that are critical to the regeneration of myelin and oligodendrocytes.

Our findings show alterations in genes involved in such diverse functions such as cell division, signaling, transcription, and development, although the largest category of genes upregulated during both demyelination and remyelination relates to inflam- 
mation and the immune response. A number of candidate genes that may be involved in the inflammation-driven regeneration of oligodendrocytes are downstream of TNF $\alpha$ during successful remyelination. We chose major histocompatibility complexes (MHCs) I and II for further analysis because the expression patterns of both were enhanced significantly during remyelination and altered by TNF $\alpha$. Furthermore, both MHC-I and -II are known to be present in demyelinating and remyelinating plaques of patients with multiple sclerosis, (Olsson, 1992).

\section{Materials and Methods}

Mice. C57BL/6J control mice were purchased from Jackson Laboratories (Bar Harbor, ME). MHC-I-deficient B2 $\mathrm{m}^{-1-}$ mice $\left(B 2 \mathrm{~m}^{\text {tmIUnc }}\right)$ were purchased from Jackson Laboratories, previously backcrossed 11 times to a C57BL6/J background (Koller et al., 1990). MHC-II ${ }^{-1-}$ mice (lacking $\mathrm{A} \beta$ ) were bred in-house and backcrossed eight times to a C57BL6/J background (Grusby et al., 1991). TNF $\alpha^{-1-}$ mice were backcrossed nine times to a C57BL6/J background at Memorial Sloan-Kettering Cancer Center and maintained in our animal facility at University of North Carolina for testing (Marino et al., 1997). All animal procedures were conducted in pathogen-free conditions with complete compliance to the National Institutes of Health Guide for the Care and Use of Laboratory Animals and were approved by the Institutional Animal Care and Use Committee of the University of North Carolina at Chapel Hill.

Induction of demyelination and remyelination. To induce demyelination, male mice, $8-10$ weeks old, were fed a diet of milled Purina mouse chow containing $0.2 \%$ cuprizone (Sigma, St. Louis, MO) for up to 6 weeks. Remyelination was initiated by returning the mice to a normal diet after 6 weeks of cuprizone (Morell et al., 1998; Matsushima and Morell, 2001).

RNA isolation. Total RNA was isolated from a dissected region of the corpus callosum of wild-type and TNF $\alpha^{-1-}$ mice at several points in treatment. RNA isolation was performed using the Qiagen RNeasy kit under RNase-free conditions (Qiagen, Valencia, CA). Purity was determined by A260/A280, by denaturing agarose gel electrophoresis, and by negative results after PCR for genomic contamination.

Microarray analysis. To profile gene expression differences, we used Affymetrix GeneChip arrays in which sets of oligonucleotides are immobilized on a chip and then hybridized with labeled RNA. These experiments used the mouse genome U74Av2 chips containing gene probes for $\sim 6000$ genes in the Mouse Unigene database as well as $\sim 6000$ expressed sequence tags clusters. cDNA synthesis was performed with the Superscript II system (Invitrogen/BRL, Grand Island, NY) and in vitro transcription labeling with biotinylated UTP and CTP was performed according to the manufacturer's recommendations (Enzo Diagnostics, Farmingdale, NY). Amplified cRNA was purified on an affinity column (Qiagen), and the quality of the amplification was verified by denaturing agarose gel electrophoresis. cRNAs were fragmented and then hybridized to prewetted array chips. Chips were washed according to Affymetrix washing protocols and subsequently stained using a fluorochrome-avidin conjugate. The probe arrays were scanned with the GeneChip system confocal scanner (Affymetrix, Santa Clara, CA). For an individual gene, hybridization of cRNA to a set of perfectly matched oligonucleotide sequences versus hybridization to a set of single mismatch oligonucleotide sequences yields a signal that is reflective of the level of expression of that gene. Data generated were analyzed using Genespring software. To lend greater fidelity to the data, this experiment was repeated, each with an RNA pool of three mice per strain and over the three chosen time points.

Quantitative real-time RT-PCR. TaqMan 5' nuclease real-time PCR assays were performed using an ABI Prism 7900 sequence-detection system (PE Applied Biosystems, Foster City, CA) in a $15 \mu$ reaction with universal master mix (Life Technologies/ BRL), $200 \mathrm{~nm}$ target primers, and $100 \mathrm{~nm}$ probe. Primers were designed to span intron-exon junctions to differentiate between cDNA and genomic DNA. The primers and probe used to detect mouse MHC-II (IA of b haplotype) were as follows: 5' primer, GAGCATCCCAGCCTGAAGA; 3' primer, CGATGCCGCTCAACATCTT; probe, Fam-ACTCAGACTGTGCCCTC CACTCCATamra. The primers and probe for mouse MHC-I (H2D of b haplotype)

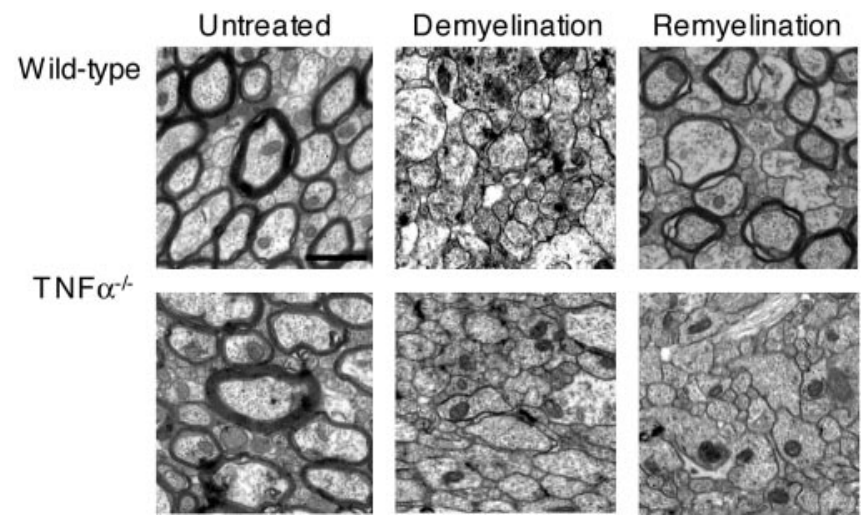

Figure 1. TNF $\alpha$ in remyelination. Electron microscopy was performed on cross-sections of the corpus callosum in wild-type and TNF $\alpha^{-1-}$ mice. Analysis is shown in untreated mice, after complete demyelination with the cuprizone, and after 2 weeks of remyelination. Scale bar, $2 \mu \mathrm{m}$.

were 5' primer, GCTCCTCACTTCCACACTGAGA; 3' primer, GGAAGAGCAGTCAGCGCTAGA; probe, Fam-AATAATTTGAATGTGGGTGGCTGGAGAGATG-Tamra. The primers and probe for mouse $18 \mathrm{~S}$ ribosomal RNA were 5' primer, GCTGCTGGCACCAGACTT; 3' primer, CGGCTACCACATCCAAGG; probe, Tet-CAAATTACCCACTCCCGACCCG-Tamra. Thermal cycle parameters were optimized to $2 \mathrm{~min}$ at $50^{\circ} \mathrm{C}$, $2 \mathrm{~min}$ at $95^{\circ} \mathrm{C}$, and 40 cycles comprising denaturation at $95^{\circ} \mathrm{C}$ for $15 \mathrm{sec}$ and annealing-extension at $56^{\circ} \mathrm{C}$ for $1.5 \mathrm{~min}$. Reactions for $18 \mathrm{~S}$ were performed during each experiment and used to normalize for amounts of cDNA. Presented results are representative of three separate trials, each performed in duplicate.

Histology and electron microscopy. Animals were prepared for frozen, paraffin, and electron microscopy (EM) sections as described previously (Arnett et al., 2001). Paraffin sections were stained with luxol-fast blue (LFB)/periodic acid Schiff for myelin, glutathione S-transferase (GST-П) for mature oligdodendrocytes (Biotrin, Newton, MA; 1:500), RCA-1 for microglia (Vector, Burlingame, CA; 1:500), and GFAP for astrocytes (Dako, Carpinteria, CA; 1:100). We quantified immunopositive cells by counting positive cells within the median of the corpus callosum, confined to a $0.033 \mathrm{~mm}^{2}$ area. Only those stained cells with an observable nucleus by DAPI stain or light microscopy were counted. Cell counts are presented as averages from at least six mice per time point. Frozen sections were stained for MHC-I (TIB126; American Type Culture Collection, Manassas, VA; 1:100) and MHC-II (PharMingen, San Diego, CA; $1: 10)$ as well as the above antibodies for colocalization. EM ultrathin sections were stained with uranyl acetate and lead citrate as described (Coetzee et al., 1996).

Statistics. Data are expressed as mean $\pm \mathrm{SD}$. Comparisons were statistically evaluated using ANOVA followed by a Bonferonni post hoc analysis. Results were considered significant if $p<0.05$.

\section{Results}

\section{Remyelination in mice lacking TNF $\alpha$}

The use of cuprizone, a copper chelating agent delivered through the diet, causes demyelination that is predictable in pathology and time course. Removal of cuprizone for 1 week causes detectable remyelination that progresses with time. Using this model, we confirmed previous findings that the degree of myelination in the corpus callosum of $\mathrm{TNF} \alpha^{-1-}$ and wild-type mice before cuprizone treatment is indistinguishable, as is their degree of demyelination and oligodendrocyte loss after an extended 5 weeks of cuprizone treatment (Arnett et al., 2001). Wild-type mice, however, underwent rapid remyelination after removal of cuprizone, whereas $\mathrm{TNF} \alpha^{-/-}$mice failed to remyelinate effectively (Fig. 1) (Arnett et al., 2001). 


$\begin{array}{lccll} & \\ \text { Cell Cycle or Proliferation-associated } \\ \text { AF061503 } & 1 & -3 & \text { block of proliferation 1 (BOP1) } \\ \text { AW048937 } & 5 & 1 & \text { Cdknla } \\ \text { M86183 } & 37 & 29 & \text { Cyclin D3 } \\ \text { X75888 } & 1 & -74 & \text { Cyclin E } \\ \text { X54149 } & 3 & 7 & \text { GADD45; MyD118 } \\ \text { J03482 } & 3 & 2 & \text { histone H1 } \\ \text { AF022110 } & \text { AP } & \text { AP } & \text { Integrin beta-5 } \\ \text { AV314625 } & 2 & 3 & \text { POLA2; DNA polymerase alpha } \\ & & & \\ \text { Developmental } & & & \\ \text { D88792 } & -3 & 1 & \text { CRP2, double LIM protein-1 } \\ \text { AV361541 } & 1 & -4 & \text { Crygd } \\ \text { X76292 } & 1 & -3 & \text { desert hedgehog } \\ \text { U13370 } & -8 & 1 & \text { Edg2; Lysophosphatidic acid receptor } \\ \text { AI842277 } & 4 & -3 & \text { IGF binding protein 3 } \\ \text { AJ005621 } & 2 & 4 & \text { LIM domain binding 3 (LDB3/Cypher) } \\ \text { M34094 } & 5 & 1 & \text { Midkine; retinoic acid-responsive protein } \\ \text { Z49086 } & 1 & 4 & \text { mouse developmental kinase 5 (MDK5) } \\ \text { Al841137 } & 7 & -5 & \text { Plexin B2 } \\ \text { AB011030 } & 1 & -3 & \text { PRDC; BMP antagonist } \\ \text { U52824 } & 2 & 3 & \text { rd5, tubby protein } \\ \text { AA866668 } & 2 & 3 & \text { Sox3 } \\ \text { AF053756 } & 7 & 4 & \text { UNC-51-like kinase (ULK1) } \\ \text { AV246963 } & -6 & 1 & \text { Wnt1 responsive Cdc42 (Wrch1) } \\ & & & \end{array}$

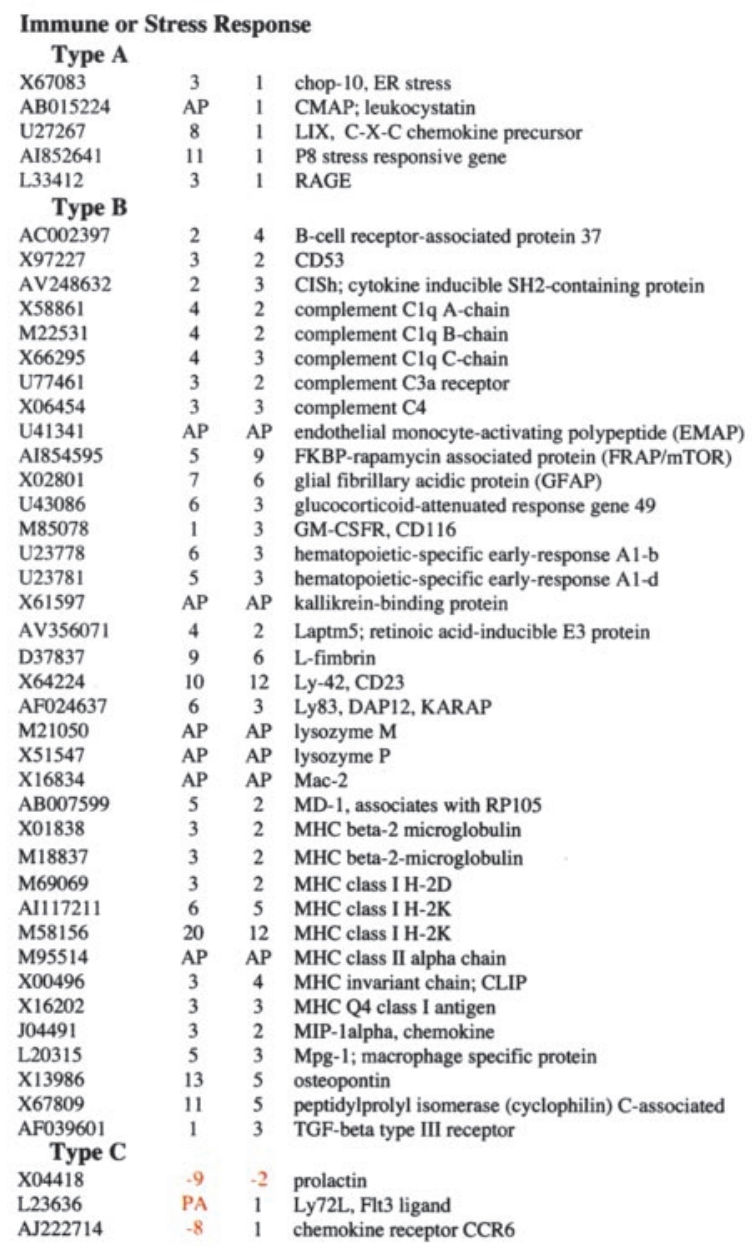

Lipid metabolism and transport
Z22661
AA770736

\section{Neuronal/Neurotransmission}

\begin{tabular}{|c|c|c|c|}
\hline L42293 & 3 & 3 & acyl-coenzyme A: cholesterol acyltransferase \\
\hline AB005450 & -4 & 1 & carbonic anhydrase XIV (CA XIV) \\
\hline AF019566 & 2 & PA & hypocretin \\
\hline D10651 & 3 & 4 & NMDAR2B \\
\hline X72966 & AP & AP & Rab3A \\
\hline X84239 & 3 & 5 & Rab5B \\
\hline Al430879 & 1 & 5 & steroid sulfatase \\
\hline AJ002306 & 3 & 3 & synaptogyrin $\mathrm{lb}$ \\
\hline AV356394 & 1 & -5 & syntrophin-1 \\
\hline \multicolumn{4}{|l|}{ Signaling } \\
\hline AF078112 & -3 & -11 & calcium signal-modulating ligand (Calmg) \\
\hline $\mathrm{X} 14836$ & 3 & 3 & CAMKII alpha \\
\hline $\mathrm{X} 87142$ & 4 & 4 & CAMKII alpha \\
\hline AF044602 & 1 & PA & G protein-coupled receptor 66 \\
\hline Y15798 & 1 & -3 & G protein-coupled receptor kinase 6 (GRK6-B) \\
\hline U02313 & -2 & -4 & MAST205 \\
\hline AB015978 & 10 & 2 & oncostatin $\mathrm{M}$ receptor beta \\
\hline M55561 & 6 & 3 & phosphatidylinositol-linked antigen (pB7) \\
\hline AV159979 & -2 & -8 & Pip5k2a; Phosphatidylinositol-4-phosphate 5-kinase \\
\hline Al195478 & 1 & -18 & Pnkp; Polynucleotide kinase 3'- phosphatase \\
\hline AW122076 & -30 & -4 & Ppplrla; Protein phosphatase inhibitor \\
\hline AV354894 & -2 & -3 & Prkm8ip; protein kinase \\
\hline AF020526 & 1 & -3 & SH2-B PH domain containing signaling mediator 1 \\
\hline D88187 & -48 & -18 & PTPf, tyrosine phosphatase \\
\hline AB014485 & 22 & 6 & RA70; src kinase-associated phosphoprotein \\
\hline \multicolumn{4}{|l|}{ Structural } \\
\hline M12481 & -2 & -3 & beta-actin \\
\hline U28932 & 1 & -4 & calponin \\
\hline AB012042 & 1 & -4 & keratin 6 beta \\
\hline AF067180 & 3 & 2 & Kif5c; kinesin heavy chain \\
\hline U96723 & 1 & 3 & myosin I beta \\
\hline AJ005567 & 4 & 4 & SPRR2I \\
\hline X53753 & PA & -2 & tropomyosin 5 \\
\hline
\end{tabular}

Transcription or Translation

$\begin{array}{lccl}\text { X00686 } & -3 & -5 & \text { 18S ribosomal RNA } \\ \text { U75530 } & 1 & -3 & \text { 4E-BP2, PHAS-II } \\ \text { AB012276 } & 14 & 1 & \text { ATFx } \\ \text { X61800 } & 4 & 2 & \text { c /ebp delta } \\ \text { AB025015 } & 4 & 3 & \text { elongin A } \\ \text { X15763 } & 1 & 3 & \text { GATA-1, DNA-binding protein } \\ \text { X98207 } & 3 & 1 & \text { Histone deacetylase 1 (HDAC1) } \\ \text { AF074882 } & 1 & 3 & \text { Histone deacetylase 3 (HDAC3) } \\ \text { U57051 } & 3 & 3 & \text { Hoxb13 } \\ \text { U20735 } & 3 & 3 & \text { junB } \\ \text { L27453 } & 2 & 3 & \text { PBX1B } \\ \text { U37500 } & -4 & 1 & \text { RNA polymerase II largest subunit } \\ \text { U21226 } & \text { AP } & \text { AP } & \text { Thing1/Hand1 } \\ \text { AB020495 } & 2 & 3 & \text { Vax1 }\end{array}$




\begin{tabular}{|c|c|c|c|c|c|c|c|}
\hline \multicolumn{4}{|c|}{ Cell Cycle or Proliferation-associated } & \multicolumn{4}{|c|}{ Neuronal/Neurotransmission } \\
\hline U90267 & -3 & 1 & Cdk $5 \mathrm{r} 2$; cyclin-dependent kinase & AV356394 & 1 & 4 & alpha1-syntrophin \\
\hline AF044336 & 1 & PA & p16, Cdkn2a & AF039833 & 2 & 4 & Caspr; neurexin IV \\
\hline X75888 & -2 & 38 & Cyclin E & AF053724 & .4 & 1 & endobrevin; VAMP-8 \\
\hline AV291335 & -2 & -4 & MCM5, replication licensing factor & AF098633 & PA & -4 & GLUT4 vesicle protein; vp115 \\
\hline AV130885 & -5 & 1 & Nap114; Nucleosome assembly protein 1-like 4 & X72966 & PA & PA & Rab3A \\
\hline AV314625 & -3 & -4 & POLA2; DNA polymerase alpha & D45207 & 2 & 3 & syntaxin 1B \\
\hline D13546 & -6 & 1 & POLA2; DNA polymerase alpha & AI326966 & -4 & 1 & urotensin II receptor \\
\hline U53584 & -3 & 1 & POLG; DNA polymerase gamma & Signaling & & & \\
\hline \multicolumn{4}{|c|}{ Developmental } & L12367 & 9 & 5 & adenylyl cyclase-associated protein (CAP) \\
\hline AV361541 & -6 & 10 & Crygd & AV220336 & -2 & -3 & cGMP-dependent protein kinase anchor (GKAP42) \\
\hline AV360936 & -11 & 1 & Crybb2; betaB2-crystallin & M94450 & -2 & -3 & EGFR-binding protein GRB7 \\
\hline U57720 & AP & AP & Cryptic; EGF-related & U49853 & 1 & -6 & FLP1, HSCF; tyrosine phosphatase \\
\hline U24160 & 1 & PA & dishevelled 2 & U87965 & 1 & -5 & G-protein 1 \\
\hline Y11245 & 1 & PA & forkhead box M1 (Foxm1) & U85714 & 1 & 3 & phospholipase C-beta-1b \\
\hline AF054623 & 6 & 1 & Frizzled-1 & AV249638 & 1 & 3 & Phosphorylase B kinase gamma catalytic chain \\
\hline X99104 & -3 & -18 & Gli2 & AV367375 & -3 & PA & PKCzeta \\
\hline AF077659 & 1 & -3 & homeodomain-interacting protein kinase 2 (Hipk2) & AA408341 & -6 & 5 & PLA2g5; secretory phospholipase \\
\hline X04480 & 3 & 5 & insulin-like growth factor I & AF030131 & -4 & 2 & Plenty of SH3s (POSH) \\
\hline X71922 & 1 & 3 & insulin-like growth factor II & AF036535 & 1 & -3 & Prkaac; AMP activated protein kinase \\
\hline U15159 & 1 & -20 & Lim kinase 1 & AF020526 & 1 & 3 & SH2-B PH domain containing signaling mediator 1 \\
\hline Z27410 & 4 & 5 & Lim homeobox protein 1 ( Lim1) & AI646422 & 1 & -3 & RIBP \\
\hline X74040 & PA & PA & forkhead box $\mathrm{C} 2$ (Foxc2) & & & & \\
\hline D88792 & 3 & 3 & CRP2, double LIM protein-1 & \multicolumn{4}{|l|}{ Structural } \\
\hline U71125 & 1 & 3 & Mesp2, Notch signaling pathway & AJ130783 & 4 & 32 & $\mathrm{APC} 2$ \\
\hline AF016714 & -2 & -3 & myeloid elf-1-like factor & U28932 & 1 & 10 & calponin; actin-binding protein \\
\hline D86948 & -3 & -3 & plexin 1 & $\mathrm{AB} 012042$ & 1 & 4 & keratin 6 beta \\
\hline U52824 & 1 & -3 & rd5; tubby protein & AJ005567 & -2 & -4 & Small proline-rich protein $2 \mathrm{i}$ \\
\hline X97818 & 4 & 1 & semaphorin $\mathbf{G}$ & X53753 & AP & 2 & tropomyosin 5 \\
\hline AA866668 & 1 & -3 & Sox 3 & & & & \\
\hline U61970 & -3 & 1 & Wnt10b & \multicolumn{4}{|c|}{ Transcription or Translation } \\
\hline \multirow[t]{2}{*}{ AV246963 } & 1 & -4 & Wnt1 responsive Cdc42 (Wrchl) & $\mathrm{X} 15763$ & 1 & -4 & GATA-1 \\
\hline & & & & AB000096 & -4 & 3 & GATA-2 \\
\hline \multicolumn{4}{|c|}{ Immune or Stress Response } & X83733 & -3 & PA & Gnrpx gene; splicing factor $3 \mathrm{a}$ \\
\hline AA050273 & 5 & 4 & CCR11, chemokine receptor & X98207 & -3 & PA & Histone deacetylase 1 (HDAC1) \\
\hline AJ222714 & 1 & -3 & CCR6 gene; chemokine receptor & AF074882 & -3 & -2 & Histone deacetylase 3 (HDAC 3 ) \\
\hline M28240 & 1 & -9 & CD19 & Y11091 & 2 & PA & map kinase interacting kinase (Mnk1) \\
\hline U60473 & -2 & -3 & CD59; complement inhibitory protein & Y11092 & 2 & -10 & map kinase interacting kinase (Mnk2) \\
\hline $\mathrm{X} 77952$ & 1 & -5 & CD105; endoglin & AF004294 & 1 & 4 & myelin transcription factor 1 (myt1) \\
\hline J04946 & 1 & 3 & CD143; angiotensin converting enzyme & $\mathrm{U} 75530$ & 1 & 4 & PHAS-II \\
\hline AF042158 & 1 & 3 & CIITA form III & L03215 & 1 & -4 & PU.1, Spi-1 \\
\hline M13926 & 3 & 2 & granulocyte colony stimulating factor 3 & U37500 & 4 & 2 & RNA polymerase II largest subunit \\
\hline AI132585 & 6 & 9 & complement $\mathrm{Cl}$ receptor & Al853032 & 1 & 5 & Sfrs4 splicing factor \\
\hline AV272809 & 1 & -4 & Dhodh & $\mathrm{U} 21226$ & -3 & PA & Thing $1 /$ Hand 1 \\
\hline AI854595 & -48 & -2 & FKBP-rapamycin associated protein (FRAP/mTOR) & & & & \\
\hline AV233170 & -4 & 7 & IFI4 interferon-activatable protein 204 & & & & \\
\hline X01973 & 1 & 8 & IFN-alpha 4 & & & & \\
\hline U78299 & 1 & -6 & lipoxin A4 receptor & & & & \\
\hline L23636 & AP & 2 & Ly72L, Flt 3 ligand & & & & \\
\hline L38423 & 1 & -3 & Lymphotoxin-beta receptor & & & & \\
\hline AB023418 & -4 & -2 & MCP-2; cytokine & & & & \\
\hline D 90146 & -4 & -2 & MHC class I Qa-2 cell surface antigen & & & & \\
\hline M27134 & -23 & -3 & MHC class I; H-2K & & & & \\
\hline M95514 & 1 & -5 & MHC class II alpha chain & & & & \\
\hline M35247 & -2 & -3 & MHC class II, T region locus 17 & & & & \\
\hline $\mathrm{X} 12760$ & 3 & 1 & Perforin & & & & \\
\hline AF030001 & 1 & -3 & RAGE & & & & \\
\hline L33412 & -2 & 3 & RAGE & & & & \\
\hline X57796 & AP & 1 & TNFR1 & & & & \\
\hline
\end{tabular}

Affymetrix gene expression data from corpus callosum of wild-type mice were compared to that of TNF $\alpha^{-1-}$ mice with similar treatments. The data are shown for TNF $\alpha^{-1-}$ mice as fold changes above or below (shown in red as a negative value) their counterpart wild-type mice. Only those genes with a raw value of $>1000$ in at least one condition, an approximately threefold change in expression, and SE of $<0.4$ of the normalized values in duplicate experiments were included for analysis. AP denotes that the expression of that gene was absent in untreated samples and present in treated samples; PA denotes that a gene went from present in untreated samples to absent in treated samples. Genes are grossly categorized according to their function, with genes of unknown function or functions other than those listed available as supplemental data (available at www.jneurosci.org).

\section{Transcripts altered in demyelinated and} remyelinating lesions

To profile gene expression differences, we used Affymetrix GeneChip arrays in which sets of oligonucleotides were immobilized on a chip and then hybridized with labeled cDNA. Three time points were chosen: untreated, 5 weeks of cuprizone treatment (representing the complete demyelination of the corpus callosum), and 6 weeks of treatment followed by 1 week of recovery ( 7 weeks; representing active remyelination). Data were analyzed in duplicate by comparison-based analysis. Only those genes with a raw value of $>1000$ in at least one condition and a greater than threefold change in expression over control were included for analysis. The overall correlation coefficient between replicate samples ranged from 0.5 to 0.8 , and for an individual gene to be included for analysis, the SE between replicates was required to be $<0.4$ of the normalized values. These relatively strict standards are likely to generate false-negative signals but less likely to produce false-positive signals. 
As expected, demyelination and oligodendrocyte apoptosis lead to the rapid downregulation of oligodendrocyte- and myelin-related genes, as shown through the gene array analysis (Table 1). These include myelin-oligodendrocyte glycoprotein (MOG), myelin-associated glycoprotein (MAG), myelin proteolipid protein (PLP), and myelin- and lymphocyte-specific protein (MAL). Genes involved in diverse functions such as cell cycle, development, and signal transduction constitute large groups of altered genes; however, the largest category of genes altered during both demyelination and remyelination consists of inflammatory and stress-response genes (Table 1). The data underscore the massive inflammatory response mounted during demyelination and remyelination. Some of these include GFAP, a marker for astrogliosis, and markers for microglia-macrophage, which have been documented to be upregulated during cuprizone treatment (Hiremath et al., 1998). The two largest components of immunerelated genes that were induced after demyelination are $\mathrm{MHC}$ and complement-related genes. Cytokines, cytokine receptors, and other inflammatory mediators were enhanced. Multiple enzymes involved in lysosomes including cathepsins $\mathrm{C}, \mathrm{H}$, and $\mathrm{Z}$ and lysoszymes $\mathrm{M}$ and $\mathrm{P}$ were also upregulated. These proteolytic enzymes may be related to the increased microglia-macrophage phagocytosis of the disrupted myelin or to the processing of antigens by MHC-I and -II. Lysozymes are often considered markers of activated cells of monocytic origin and have been shown previously to correlate with the recruitment of microglia-macrophages after demyelination (Morell et al., 1998; Jurevics et al., 2002).

Two primary patterns of gene expression are observed among the immune genes shown in Table 1 . In the group labeled Type A, the genes were upregulated during demyelination but then reversed to basal levels. In the group labeled Type B, the genes were upregulated during both demyelination and remyelination. Remarkably, most of the immune response genes belong to the latter. This suggests that the immune transcripts upregulated during remyelination do not represent a subset of the immune response but rather a more generalized effect. It also implicates inflammatory genes as important components of remyelination.

\section{Transcripts altered in mice lacking TNF $\alpha$}

To identify genes that are involved in remyelination, it is important to compare gene expression profiles of mice that have undergone successful remyelination with those that have exhibited unsuccessful or delayed remyelination. The results with TNF $\alpha^{-1-}$ mice (Fig. 1) indicate that a comparison of these mice with wild-type mice during the remyelination phase should reveal genes that are putatively important for remyelination. Affymetrix analyses of cDNA from the corpus callosum of these two sets of mice were performed at the following time points: before cuprizone treatment, when demyelination was completed in both strains ( 5 weeks of cuprizone treatment), and when remyelination was substantial in the wild-type mice, but delayed in the $\mathrm{TNF} \alpha^{-1-}$ mice (at 7 weeks, when cuprizone was removed for the last week).

In agreement with the histologic and pathologic analysis, $\mathrm{TNF} \alpha^{-1-}$ and wild-type mice showed few differences in gene expression before cuprizone treatment (data not shown). In contrast, significant differences were found between $\mathrm{TNF} \alpha^{-1-}$ and wild-type mice after demyelination as well as during remyelination (Table 2). Because TNF $\alpha^{-1-}$ mice showed defective remyelination at the week 7 time point, special emphasis was placed on these gene differences. The primary categories of genes that were expressed more highly in wild-type mice than $\mathrm{TNF} \alpha^{-1-}$ mice

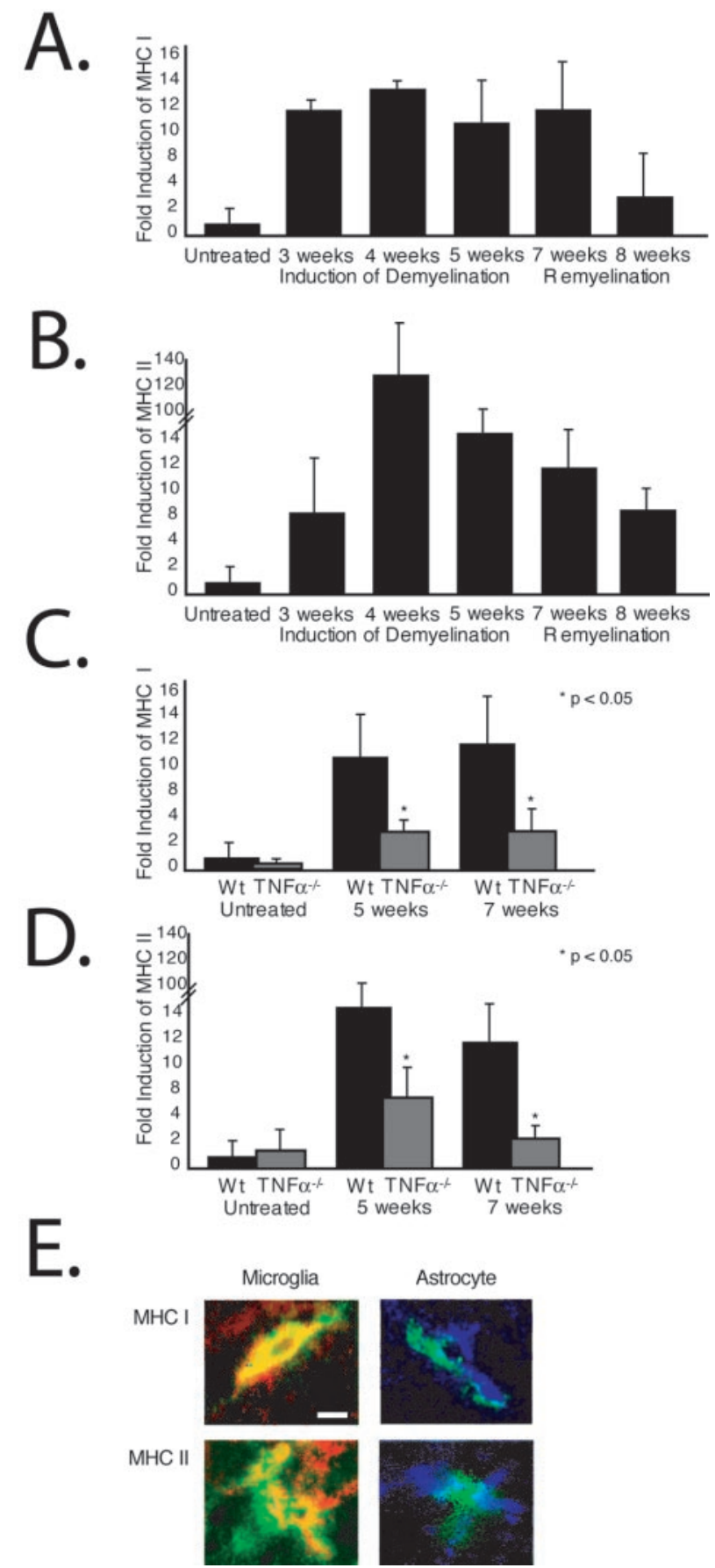

Figure 2. Expression of MHC-I and -II. A, B, Quantitative analysis of MHC-I and -II transcripts using quantitative real-time RT-PCR on total RNA isolated from the corpus callosum during demyelination and remyelination in wild-type mice. Each bar represents the averaged fold induction over untreated wild-type mice of at least three mice per genotype per time point $( \pm S D)$. The scale is interrupted to accommodate the large value obtained for MHC-II with wild-type mice after 4 weeks of cuprizone treatment. C, D, Quantitative analysis of MHC-I and -II transcripts in wild-type mice compared with that in mice lacking TNF $\alpha$. ${ }^{*} p<0.05$. E, Antibodies were used to localize expression of MHC-I and -II (green) during demyelination of wild-type mice and colocalized to microglia (RCA-1, red) and astrocytes (GFAP, blue). Yellow indicates colocalization of the red and green fluorochromes. Scale bar, $12.5 \mu \mathrm{m}$.

during the remyelinating phase include immune response, signaling, and developmental and transcriptional regulation (Table 2). Notably several genes in the MHC family were reduced in mice lacking TNF $\alpha$. 
A.
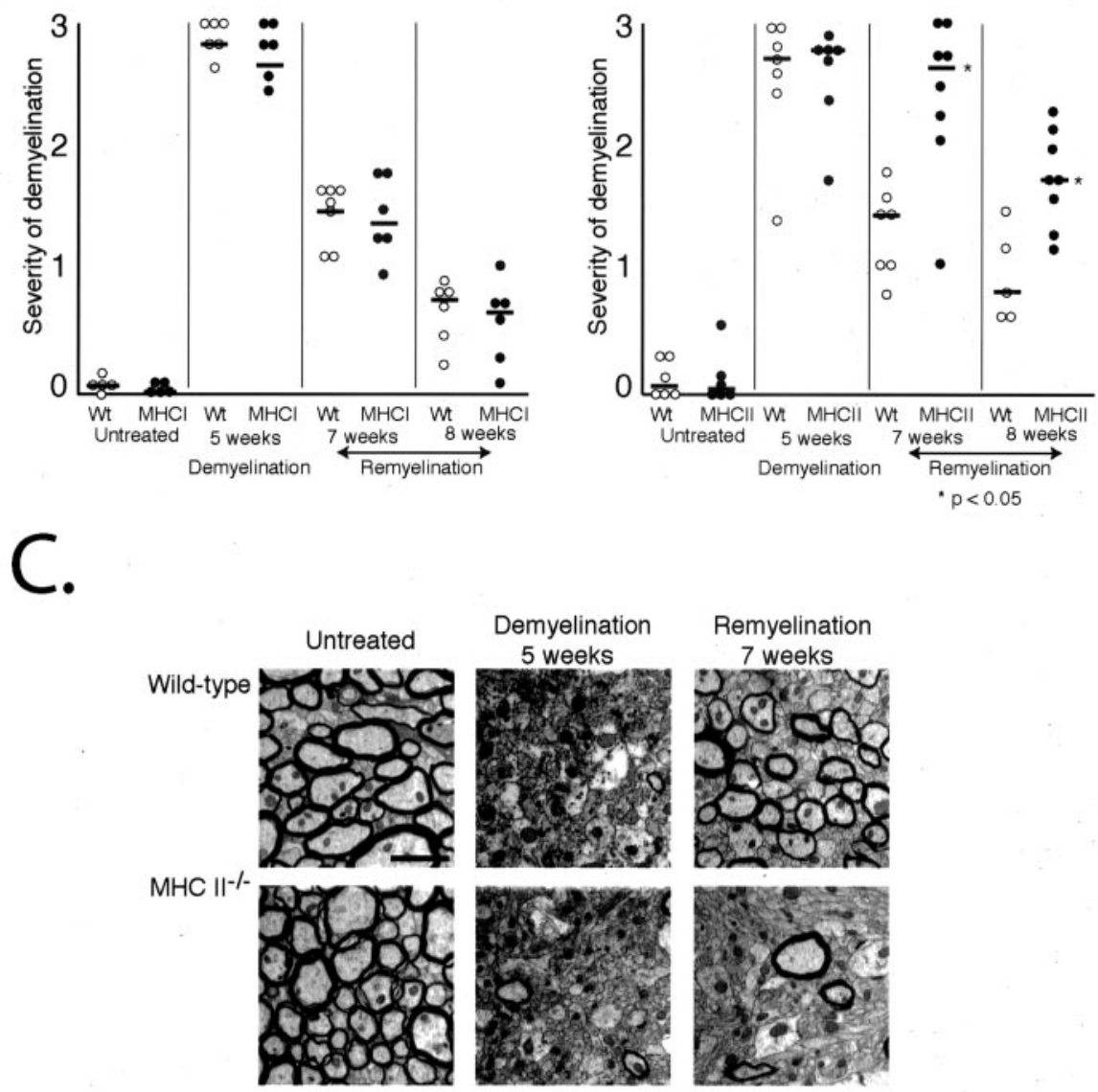

D.

$\begin{gathered}\text { Untreated } \\ \text { Wild-type } \\ \text { MHC } \|^{-/-}\end{gathered}$
Treated for 5 weeks
Wild-type
MHC $\|^{-/-}$
Treated for 6 weeks
+1 week recovery
Wild-type
MHC ${ }^{-/-}$
${ }^{*} p<0.001$

\begin{tabular}{cc} 
g-ratio & Percent unmyelinated fibers \\
\hline $0.717+/-0.032$ & $0.6 \%$ \\
$0.748+/-0.014$ & $0.5 \%$
\end{tabular}

$0.973+/-0.078 \quad 88.6 \%$

$0.941+/-0.062 \quad 88.0 \%$

$0.816+/-0.036 \quad 31.5 \%$

$0.923+/-0.042^{*} \quad 78.0 \%{ }^{*}$

${ }^{*} p<0.001$

Figure 3. $\mathrm{MHC}$-I and-II in remyelination. A, B, Wild-type mice, MHC-I-null mice, and MHC-II-null mice were analyzed at various time points during demyelination and remyelination. Sections containing the corpus callosum at the level of the fornix were stained for LFB/PAS, and the corpus callosum was scored by three investigators on a scale of 0 (completely myelinated) to 3 (completely demyelinated), with the presence of blue fibers indicative of intact myelin. ${ }^{*} p<0.05$. C, Remyelination was examined further with electron microscopy. Pictures represent a cross-section of the corpus callosum at the level of the fornix in untreated mice, mice completely demyelinated with cuprizone, and mice allowed to remyelinate for 1 week. Scale bar, $2 \mu \mathrm{m}$. $D$, $g$-ratios and percentage unmyelinated fibers were calculated from a minimum of 200 fibers per mouse per time point. ${ }^{*} p<0.001$.

\section{Expression of MHC-I and -II during demyelination and remyelination}

The considerable number of MHC-related genes that were enhanced during demyelination and remyelination in wild-type mice and their alteration by the absence of TNF $\alpha$ implicate a role for these genes in the control of remyelination. We verified expression of MHC-I and -II in this disease model through quan- titative real-time RT-PCR at time points additional to those included in the Affymetrix analysis. This analysis shows similar patterns of increases in the transcription of MHC-I and -II during cuprizoneinduced inflammation, with the peak of expression occurring at 4 weeks of cuprizone treatment (Fig. 2A,B). This time point correlates with the peak of microgliamacrophage infiltration into the corpus callosum (Hiremath et al., 1998). Elevated MHC-I and -II expression was maintained during remyelination, although the level decreased at the 8-week endpoint. Levels of MHC-I and -II were decreased after demyelination and remyelination in the absence of TNF $\alpha(p<0.05)$ (Fig. 2C,D), although the degree of microglia-macrophage recruitment was similar in the wildtype and $\mathrm{TNF} \alpha^{-/-}$mice in this model (Arnett et al., 2001). Thus, transcription of both MHC-I and -II genes is induced by $\mathrm{TNF} \alpha$, but $\mathrm{TNF} \alpha$ is not required for MHC gene expression because expression is still observed in $\mathrm{TNF} \alpha^{-1-}$ mice. Detectable MHC-I (Fig. 2 E, green) predominantly localizes to both microglia (red) and astrocytes (blue) in the demyelinating lesion. MHC-II is expressed primarily on microglia but also occasionally on astrocytes (Fig. 2E).

Roles of MHC-I and -II in remyelination To perform functional genomic analysis, we characterized the roles of MHC-I and -II in remyelination using mice lacking expression of MHC-I or -II. The mice used to study the former lack B2m, which is essential for the expression of properly folded MHC-I protein (Koller et al., 1990). The mice used to study the latter lacked $\mathrm{A} \beta$ chain, which eliminates I-A expression (Grusby et al., 1991). Because of a natural defect in the E $\alpha$ promoter of MHC II, this strain lacks all MHC-II expression. Untreated wild-type and MHC-I or -II null mice exhibited similar degrees of myelination before treatment (Fig. $3 A, B$, untreated). The degree of demyelination was determined by the extent of LFB staining and was read in a double-blind manner by three investigators. All three strains were also completely demyelinated after the extended 5 weeks of cuprizone treatment (Fig. $3 A, B, 5$ weeks). When the toxin was removed, wild-type mice and $\mathrm{MHC}-\mathrm{I}^{-1-}$ mice exhibited rapid remyelination within 1 week, which progressed steadily by 2 weeks (Fig. $3 A, 7$ and 8 weeks). In dramatic contrast, MHC-II ${ }^{-1-}$ mice demonstrated a significant delay in remyelination $(p<0.05)$ (Fig. $3 B, 7$ and 8 weeks).

LFB analysis is a qualitatitve measurement of myelination. Ultrastructural analysis and quantitation by electron microscopic inspection represent much more accurate approaches to 
determine the extent of myelination in $\mathrm{MHC}-\mathrm{II}^{-1-}$ versus wild-type mice (Fig. $3 C)$. A measurement of myelination, the $g$-ratio, was calculated as the ratio of the diameter of the axon to the diameter of the axon and surrounding myelin. Three mice from each group at each time point were tested, and a minimum of 200 fibers per mouse per strain per time point were measured. This provides an assessment of the percentage of unmyelinated axons throughout the treatment protocol in wild-type and MHC-II-null mice (Fig. 3D). A typical $g$-ratio for a normally myelinated axon is between 0.6 and 0.8 , where a $g$-ratio of 1.0 is completely demyelinated. After 5 weeks of treatment, $88-89 \%$ of axons in the corpus callosum of wild-type and ${\mathrm{MHC}-\mathrm{II}^{-1-}}^{-1-}$ mice were completely demyelinated. Within 1 week of remyelination, $32 \%$ of wild-type axons remained demyelinated compared with $78 \%$ in $\mathrm{MHC}-\mathrm{II}^{-1-}$ mice.

During the demyelination process, mature oligodendrocytes in the corpus callosum undergo apoptosis (Mason et al., 2000). Remyelination is initiated with the reappearance of new myelinating oligodendrocytes in the lesion. The oligodendrocytes in wild-type and $\mathrm{MHC}^{-\mathrm{II}^{-1-}}$ mice were depleted from the corpus callosum to similar degrees after 5 weeks of cuprizone treatment; however, after 1 week of recovery, wild-type mice had twofold more oligodendrocytes in the corpus callosum than $\mathrm{MHC}^{-\mathrm{II}^{-/}}$mice $(p<0.05)$ (Fig. $4 A, B$ ). In contrast, the results with $\mathrm{MHC}^{-\mathrm{I}^{-}}$- mice are indistinguishable from wild-type mice. This finding suggests that MHC-II is important in the regeneration of new oligodendrocytes and explains the delay in remyelination observed in mice lacking MHC-II.
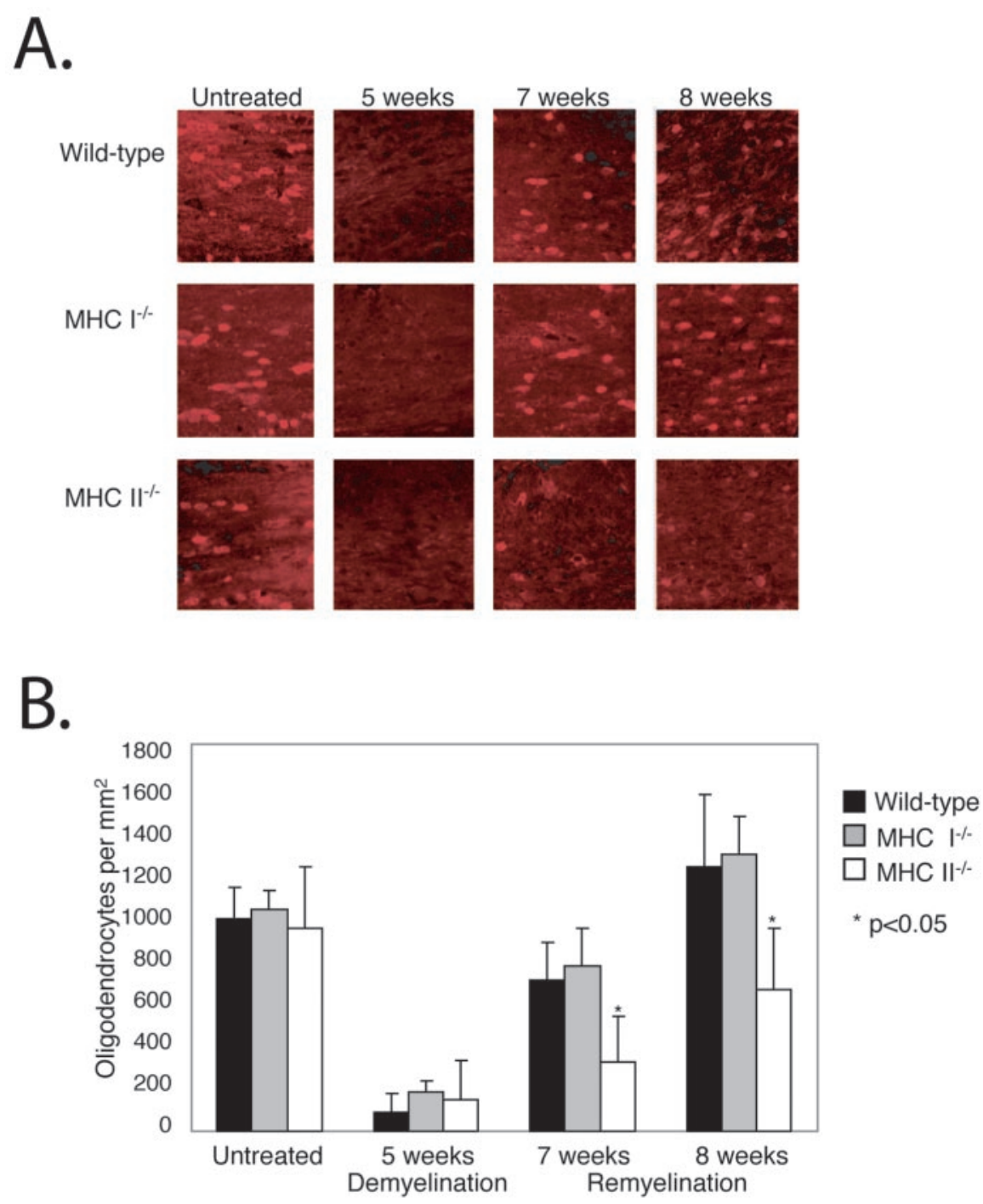

Figure 4. MHC-II and the regeneration of oligodendrocytes. $A$, Oligodendrocytes were identified during demyelination and remyelination in wild-type mice and mice lacking MHC-I or -II using an antibody to GST-П (red). B, Quantification of oligodendrocytes in the corpus callosum. Each bar represents an average of the results of at least six mice per genotype per time point. ${ }^{*} p<0.05$.

\section{Discussion}

In an effort to identify important factors in myelination, we have identified patterns of gene expression observed during cuprizone-induced demyelination and remyelination in wildtype mice. These were compared with $\mathrm{TNF} \alpha^{-1-}$ mice, which showed retarded remyelination. Two of the most impressive findings are the predominance of immune and stress response genes during both demyelination and remyelination, and the significant effects of TNF $\alpha$ on inflammatory gene expression. In particular, MHC-II is upregulated during both demyelination and remyelination, is controlled by $\mathrm{TNF} \alpha$, and is important for the reappearance of new, myelinating oligodendrocytes required for successful remyelination. Interestingly, the genes for TNF $\alpha$ and MHC-II are proximal to each other, and they represent the only consistent linkages to MS susceptibility in humans (Fukazawa et al., 2000; Kantarci et al., 2002). Further study of MHC-II and other genes identified in this screen could establish them as important and novel therapeutic targets in demyelinating diseases.

Cuprizone is an ideal model for studying demyelination, re- myelination, and the role of neuroinflammation. The induction of demyelination and remyelination is easy to administer, and the outcome is predictable in pathology and disease course. Administration of cuprizone to mice results in an impressive inflammatory reaction reflected by tremendous microglial accumulation and astrogliosis (Hiremath et al., 1998; Arnett et al., 2001; McMahon et al., 2001). In retrospect, it is not surprising that most of the genes upregulated after demyelination and during remyelination are involved in the inflammatory response. Remarkably, many of these immune response genes are related to the MHC family.

MHC-I and -II are increased under a wide range of pathologic conditions and play multiple roles during inflammation in the CNS (Neumann, 2001). In the brain, these factors have been most studied in the presentation of antigens in murine experimental autoimmune encephalitis in which demyelination follows the generation of myelin-reactive $\mathrm{T}$ lymphocytes. The presence of MHC-II has also been shown to be protective for regeneration and axon integrity in a mouse model of viral encephalitis (Njenga et al., 1999). Low levels of MHC-I are present on most cells and 
function by presenting endogenous foreign peptides to CD8+ cells. Alternatively, MHC-II is present on professional antigenpresenting cells, including macrophages and dendritic cells, and presents exogenous antigenic peptides to $\mathrm{CD} 4+$ cells. Both microglia and astrocytes express MHC-II in vitro, but microglia appear to represent the predominant MHC-II-positive cells in vivo, similar to our observations in this study (Dong and Benveniste, 2001).

Although antigen presentation is a primary function of MHC-I and -II, a T lymphocyte infiltrate is not easily found in the cuprizone model, where the blood-brain barrier remains intact (Matsushima and Morell, 2001). Furthermore, the remyelination phase is independent of $\mathrm{T}$ cells as shown by the use of RAG ${ }^{-1-}$ mice, which lack lymphocytes (Arnett et al., 2001). MHC-II has been previously hypothesized to have signaling functions in microglia and macrophages independent of $\mathrm{T}$ lymphocytes through its cytoplasmic domain. MHC-II has been demonstrated to contribute to the activation of microglia-macrophage in the brain in a model of Krabbes disease, in which lymphocytes are also not known to play a role (Matsushima et al., 1994). Future experiments will be directed at addressing the signaling potential of MHC-II in this model.

The use of gene array analysis has provided extraordinary insights regarding gene alterations during different permutations; however, the significance of functional genomics is underscored by the failure to find a role for MHC-I in the remyelination phase despite significant changes in this and related genes during demyelination, remyelination, and after TNF $\alpha$ deletion. The most straightforward possibility is that $\mathrm{TNF} \alpha$, a well known inducer of MHC-I (Panek et al., 1994; Neumann et al., 1997), obligatorily induces MHC-I, but this has no biologic consequences on the myelination process. Another possibility is that the enhanced levels of MHC-I have functions that are not revealed by our analysis. We have used mice lacking $\mathrm{B} 2 \mathrm{~m}$, which might not be the same as a deletion of the gene for MHC-I, because expression of "empty" MHC-I can be forced on cells without B2m (Allen et al., 1986; Bix and Raulet, 1992). We are aware that previous studies of transgenic mice with MHC-I expression in oligodendrocytes have observed alterations in myelination (Turnley et al., 1991). In those studies, however, the expression of MHC-I is forced and on oligodendrocytes, which do not normally express MHC.

Although it is clear that TNF $\alpha$ and MHC-II are involved in the regeneration of oligodendrocytes and remyelination, the mechanism for the precise involvement of the immune system in remyelination is unclear. One possibility is that the expression of these inflammatory molecules causes the upregulation, either directly or indirectly, of factors that contribute to the proliferation and maturation of new progenitors. This study supports that hypothesis by showing an upregulation of multiple transcription factors and other proteins with known functions in the development of the CNS after the immune response to demyelination.

In summary, this study supports the possibility that inflammation has its use in the repair of demyelination, although not all immune-related molecules that are enhanced during remyelination appear to have a direct functional impact on this process. Sorting out the inflammatory processes that are beneficial versus those that may be harmful is crucial to the design of improved therapies for demyelinating or dysmyelinating disorders.

\section{References}

Allen H, Fraser J, Flyer D, Calvin S, Flavell R (1986) Beta 2-microglobulin is not required for cell surface expression of the murine class I histocompatibility antigen $\mathrm{H}-2 \mathrm{Db}$ or of a truncated H-2Db. Proc Natl Acad Sci USA 83:7447-7451.
Arnett HA, Mason J, Marino M, Suzuki K, Matsushima GK, Ting JP (2001) TNF alpha promotes proliferation of oligodendrocyte progenitors and remyelination. Nat Neurosci 4:1116-1122.

Arnett HA, Hellendall RP, Matsushima GK, Suzuki K, Laubach VE, Sherman P, Ting JP (2002) The protective role of nitric oxide in a neurotoxicantinduced demyelinating model. J Immunol 168:427-433.

Bix M, Raulet D (1992) Functionally conformed free class I heavy chains exist on the surface of beta 2 microglobulin negative cells. J Exp Med $176: 829-834$.

Brosnan CF, Raine CS (1996) Mechanisms of immune injury in multiple sclerosis. Brain Pathol 6:243-257.

Coetzee T, Fujita N, Dupree J, Shi R, Blight A, Suzuki K, Popko B (1996) Myelination in the absence of galactocerebroside and sulfatide: normal structure with abnormal function and regional instability. Cell 86:209-219.

Dong Y, Benveniste EN (2001) Immune function of astrocytes. Glia 36:180-190.

Eugster HP, Frei K, Bachmann R, Bluethmann H, Lassmann H, Fontana A (1999) Severity of symptoms and demyelination in MOG-induced EAE depends on TNFR1. Eur J Immunol 29:626-632.

Fukazawa T, Sasaki H, Kikuchi S, Hamada T, Tashiro K (2000) Genetics of multiple sclerosis. Biomed Pharmacother 54:103-106.

The Lenercept Multiple Sclerosis Study Group (1999) TNF neutralization in MS: results of a randomized, placebo-controlled multicenter study. Neurology 53:457-465.

Grusby MJ, Johnson RS, Papaioannou VE, Glimcher LH (1991) Depletion of CD4+ T cells in major histocompatibility complex class II-deficient mice. Science 253:1417-1420.

Hiremath MM, Saito Y, Knapp GW, Ting JP, Suzuki K, Matsushima GK (1998) Microglial/macrophage accumulation during cuprizone-induced demyelination in C57BL/6 mice. J Neuroimmunol 92:38-49.

Juedes AE, Ruddle NH (2001) Resident and infiltrating central nervous system APCs regulate the emergence and resolution of experimental autoimmune encephalomyelitis. J Immunol 166:5168-5175.

Jurevics H, Largent C, Hostettler J, Sammond DW, Matsushima GK, Kleindienst A, Toews AD, Morell P (2002) Alterations in metabolism and gene expression in brain regions during cuprizone-induced demyelination and remyelination. J Neurochem 82:126-136.

Kantarci OH, de Andrade M, Weinshenker BG (2002) Identifying disease modifying genes in multiple sclerosis. J Neuroimmunol 123:144-159.

Kassiotis G, Kollias G (2001) Uncoupling the proinflammatory from the immunosuppressive properties of tumor necrosis factor (TNF) at the p55 TNF receptor level. Implications for pathogenesis and therapy of autoimmune demyelination. J Exp Med 193:427-434.

Kassiotis G, Pasparakis M, Kollias G, Probert L (1999) TNF accelerates the onset but does not alter the incidence and severity of myelin basic proteininduced experimental autoimmune encephalomyelitis. Eur J Immunol 29:774-780.

Koller BH, Marrack P, Kappler JW, Smithies O (1990) Normal development of mice deficient in beta $2 \mathrm{M}, \mathrm{MHC}$ class I proteins, and CD8+ T cells. Science 248:1227-1230.

Korner H, Riminton DS, Strickland DH, Lemckert FA, Pollard JD, Sedgwick JD (1997) Critical points of tumor necrosis factor action in central nervous system autoimmune inflammation defined by gene targeting. J Exp Med 186:1585-1590.

Liu J, Marino MW, Wong G, Grail D, Dunn A, Bettadapura J, Slavin AJ, Old L, Bernard CC (1998) TNF is a potent anti-inflammatory cytokine in autoimmune-mediated demyelination. Nat Med 4:78-83.

Locksley RM, Killeen N, Lenardo MJ (2001) The TNF and TNF receptor superfamilies: integrating mammalian biology. Cell 104:487-501.

Marino MW, Dunn A, Grail D, Inglese M, Noguchi Y, Richards E, Jungbluth A, Wada H, Moore M, Williamson B, Basu S, Old LJ (1997) Characterization of tumor necrosis factor-deficient mice. Proc Natl Acad Sci USA 94:8093-8098.

Mason JL, Jones JJ, Taniike M, Morell P, Suzuki K, Matsushima GK (2000) Mature oligodendrocyte apoptosis precedes IGF-1 production and oligodendrocyte progenitor accumulation and differentiation during demyelination/remyelination. J Neurosci Res 61:251-262.

Matsushima GK, Morell P (2001) The neurotoxicant, cuprizone, as a model to study demyelination and remyelination in the central nervous system. Brain Pathol 11:107-116.

Matsushima GK, Taniike M, Glimcher LH, Grusby MJ, Frelinger JA, Suzuki 
K, Ting JP (1994) Absence of MHC class II molecules reduces CNS demyelination, microglial/macrophage infiltration, and twitching in murine globoid cell leukodystrophy. Cell 78:645-656.

McMahon EJ, Cook DN, Suzuki K, Matsushima GK (2001) Absence of macrophage-inflammatory protein-1alpha delays central nervous system demyelination in the presence of an intact blood-brain barrier. J Immunol 167:2964-2971.

Mohan N, Edwards ET, Cupps TR, Oliverio PJ, Sandberg G, Crayton H, Richert JR, Siegel JN (2001) Demyelination occurring during antitumor necrosis factor alpha therapy for inflammatory arthritides. Arthritis Rheum 44:2862-2869.

Morell P, Barrett CV, Mason JL, Toews AD, Hostettler JD, Knapp GW, Matsushima GK (1998) Gene expression in brain during cuprizone-induced demyelination and remyelination. Mol Cell Neurosci 12:220-227.

Neumann H (2001) Control of glial immune function by neurons. Glia 36:191-199.

Neumann H, Schmidt H, Cavalie A, Jenne D, Wekerle H (1997) Major histocompatibility complex (MHC) class I gene expression in single neurons of the central nervous system: differential regulation by interferon (IFN)gamma and tumor necrosis factor (TNF)-alpha. J Exp Med 185:305-316.

Njenga MK, Murray PD, McGavern D, Lin X, Drescher KM, Rodriguez M (1999) Absence of spontaneous central nervous system remyelination in class II-deficient mice infected with Theiler's virus. J Neuropathol Exp Neurol 58:78-91.

Olsson T (1992) Immunology of multiple sclerosis. Curr Opin Neurol Neurosurg 5:195-202.

Panek RB, Lee YJ, Itoh-Lindstrom Y, Ting JP, Benveniste EN (1994) Character- ization of astrocyte nuclear proteins involved in IFN-gamma- and TNFalpha-mediated class II MHC gene expression. J Immunol 153:4555-4564.

Raine CS, Bonetti B, Cannella B (1998) Multiple sclerosis: expression of molecules of the tumor necrosis factor ligand and receptor families in relationship to the demyelinated plaque. Rev Neurol (Paris) 154:577-585.

Robinson WH, Genovese MC, Moreland LW (2001) Demyelinating and neurologic events reported in association with tumor necrosis factor alpha antagonism: by what mechanisms could tumor necrosis factor alpha antagonists improve rheumatoid arthritis but exacerbate multiple sclerosis? Arthritis Rheum 44:1977-1983.

Scherbel U, Raghupathi R, Nakamura M, Saatman KE, Trojanowski JQ Neugebauer E, Marino MW, McIntosh TK (1999) Differential acute and chronic responses of tumor necrosis factor-deficient mice to experimental brain injury. Proc Natl Acad Sci USA 96:8721-8726.

Sicotte NL, Voskuhl RR (2001) Onset of multiple sclerosis associated with anti-TNF therapy. Neurology 57:1885-1888.

Sriram S, Rodriguez M (1997) Indictment of the microglia as the villain in multiple sclerosis. Neurology 48:464-470.

Suvannavejh GC, Lee HO, Padilla J, Dal Canto MC, Barrett TA, Miller SD (2000) Divergent roles for $\mathrm{p} 55$ and p75 tumor necrosis factor receptors in the pathogenesis of MOG(35-55)-induced experimental autoimmune encephalomyelitis. Cell Immunol 205:24-33.

Turnley AM, Morahan G, Okano H, Bernard O, Mikoshiba K, Allison J, Bartlett PF, Miller JF (1991) Dysmyelination in transgenic mice resulting from expression of class I histocompatibility molecules in oligodendrocytes. Nature 353:566-569. 